\title{
The first close-up of the "flip-flop" phenomenon in a single star
}

\author{
H. Korhonen ${ }^{1}$, S. V. Berdyugina ${ }^{1}$, K. G. Strassmeier ${ }^{2}$, and I. Tuominen ${ }^{1}$ \\ 1 Astronomy Division, PO Box 3000, 90014 University of Oulu, Finland \\ 2 Astrophysikalisches Institut Potsdam, An der Sternwarte 16, 14482 Potsdam, Germany
}

Received 3 September 2001 / Accepted 1 October 2001

\begin{abstract}
We present temperature maps of the active late-type giant FK Com which exhibit the first imagining record of the "flip-flop" phenomenon in a single star. The phenomenon, in which the main part of the spot activity shifts $180^{\circ}$ in longitude, discovered a decade ago in FK Com, was reported later also in a number of RS CVn binaries and a single young dwarf. With the surface images obtained right before and after the "flip-flop", we clearly show that the "flip-flop" phenomenon in FK Com is caused by changing the relative strengths of the spot groups at the two active longitudes, with no actual spot movements across the stellar surface, i.e. exactly as it happens in other active stars.
\end{abstract}

Key words. stars: activity - imaging - individual: FK Com - late-type - starspots

\section{Introduction}

In order to study the spot evolution in cool active stars, two approaches are commonly used: photometric light curve modeling (or inversion) and Doppler imaging. The light curve analysis is evidently a much poorer source of information than Doppler imaging and, therefore, leaves more freedom for conclusions about the spot evolution. However, the amount, frequency and time scale of photometric observations exceed significantly those of Doppler images obtained to date (see the most recent compilation of Doppler imaging results given by Strassmeier 2001). Doppler images, in turn, reveal key properties of spots, such as their latitudes, actual motion, areas, etc., which could strongly constrain conclusions made from light curve modeling.

In this letter, using both techniques, we investigate a so-called "flip-flop" phenomenon, which was discovered a decade ago in light curve behaviour of a single, late-type giant FK Com (Jetsu et al. 1991, 1993, 1994). In this phenomenon, the concentrated part of the spot activity shifts $180^{\circ}$ in longitude over a short period of time and remains for some years at a new active longitude. Since its discovery, the "flip-flop" phenomenon has also been reported

Send offprint requests to: H. Korhonen,

e-mail: heidi.korhonen@oulu.fi

* Based on the observations obtained at the Kitt Peak National Observatory, USA; the Automatic Photometric Telescope, Phoenix 10, Arizona, USA; the Nordic Optical Telescope, Observatorio Roque de los Muchachos, La Palma, Canary Islands, Spain. in some RS CVn binaries and in a single young dwarf LQ Hya (Jetsu 1996; Berdyugina \& Tuominen 1998, 2001; Berdyugina et al. 1998, 1999, 2000; Rodonó et al. 2000), implying that the phenomenon is a significant pattern of the activity in different types of stars and requires a thorough investigation.

The active, late-type, giant FK Com itself is a prototype of a small group of rapidly rotating, single, giants (Bopp \& Stencel 1981). It exhibits strong and variable chromospheric and photospheric activity. Small variations in FK Com's visual magnitude were first reported by Chugainov (1966), and its spectral peculiarities include a strong and variable $\mathrm{H} \alpha$ emission (e.g. Ramsey et al. 1981), strong chromospheric UV emission (Bopp \& Stencel 1981) and high X-ray luminosity (Walter 1981).

Since in FK Com the "flip-flop" phenomenon was only observed as a shift of the light curve minimum, it was not clear whether the spots themselves move across the stellar surface. This could be only judged with the technique of Doppler imaging. The first hint came from the surface map for the year 1994 (Korhonen et al. 1999, from here-on Paper I), which seemed to present the star right at the moment of the "flip-flop", when one active region was getting weaker and another, in the opposite active longitude $180^{\circ}$ apart, was taking over. Also, photometric observations have indicated that the two active longitudes in FK Com can sometimes both be active at the same time (Jetsu et al. 1994). This implies that the "flip-flop" in FK Com is caused by rearrangement of the spot activity at the active longitudes, and not by movement of the spots themselves. 
Peculiar phase shifts of light curve minima due to "flipflops" being interpreted as movements of spots can result in variations of the spot rotation period determined by e.g. the Fourier analysis. Further, such period variations are normally interpreted as indications of the stellar differential rotation (e.g. Hall 1991; Henry et al. 1995), which can be therefore overestimated by few orders of magnitude. In FK Com, for instance, an interval of the period variations is from 2.3960 to 2.4047 (Paper I) which could indicate the differential rotation rate larger than 0.004 , while our Doppler imaging results limited it to $0.0001 \pm 0.0002$, i.e. in fact gave evidence for a rigid rotation of the stellar surface (Korhonen et al. 2000, from here-on Paper II).

The photometric behaviour of the other types of stars during the "flip-flop" phenomenon was found to be very similar to that of FK Com. Moreover, Doppler imaging results for two RS CVn binaries II Peg and IM Peg clearly demonstrated that in this type of stars the "flip-flop" is caused by diminishing spot area in one active longitude and simultaneous increasing spot area in the opposite longitude, i.e. switching the activity between the active longitudes due to relative spot area evolution rather than spot motion (Berdyugina et al. 1998, 1999, 2000). This suggests that we could expect to observe something similar in FK Com.

It was also noted that the flipping in RS CVn stars happens periodically and can be predicted with a good accuracy (Berdyugina \& Tuominen 1998; Rodonó et al. 2000). In FK Com, the full period of "flip-flops", i.e. when the activity returns to the same active longitude, was determined to be 6.5 years (Paper I). This period has been confirmed by the period analysis of 35 years of photometric observations (Korhonen et al. 2001a, 2001b, from here-on Papers III and IV). The previous "flip-flop" has occurred during the summer 1994 (Papers I, III and IV) and the next had to be expected in about 3.3 years, i.e. in the end of 1997.

In this paper, we confirm the occurrence of the "flipflop" in FK Com in the second half of 1997 and present the first temperature maps obtained right before and just after the "flip-flop". These maps clearly show that the "flipflop" in FK Com is caused by activity switching between the active longitudes without spot movement across the stellar surface, i.e. similarly to the RS CVn stars.

\section{Observations}

Two sets of spectroscopic observations of FK Com are used in this paper. The first set is from June 1997 and the details of these observations are given in Paper II. The second set was observed at the Kitt Peak National Observatory (KPNO) with the 0.9-m coudé feed telescope during 10 nights between 29th of December 1997 and 15th of January 1998. The TI- $500 \times 800$ CCD detector was employed together with grating A, camera 5 , the long collimator, and a $280-\mu \mathrm{m}$ slit to give a resolving power of 38000 at $6500 \AA$. The useful wavelength range was $80 \AA$ and the exposure time was set to $900 \mathrm{~s}$. The reductions
Table 1. Spectroscopic observations of FK Com obtained between 29th of December 1997 and 15th of January 1998 at $\mathrm{KPNO}$

\begin{tabular}{lcclcc}
\hline $\begin{array}{l}\text { HJD } \\
2450000+\end{array}$ & Phase & $S / N$ & $\begin{array}{l}\text { HJD } \\
2450000+\end{array}$ & Phase & $S / N$ \\
\hline 811.99 & 0.87 & 222 & 822.02 & 0.05 & 219 \\
814.00 & 0.71 & 291 & 823.03 & 0.47 & 273 \\
815.02 & 0.14 & 296 & 826.04 & 0.72 & 243 \\
820.02 & 0.22 & 218 & 828.04 & 0.55 & 252 \\
820.99 & 0.62 & 228 & 829.00 & 0.95 & 321 \\
\hline
\end{tabular}

were done with the Image Reduction and Analysis Facility (IRAF) distributed by KPNO/NOAO. A summary of the KPNO observations can be found in Table 1.

We also have simultaneous photometric observations in $B$ and $V$ from the Automatic Photometric Telescope (APT), Phoenix 10, Arizona, USA. For checking the reliability of the temperature map from January 1998 we have used 24 observations taken between the 28th of November 1997 and the 7th of January 1998. The observations obtained later than 7 th of January are not included because of the very rapid change of the light curve in the beginning of 1998, especially for the earlier phases for which the spectroscopic observations have been obtained 8th of January or before. Observations with mean errors larger than $0 .{ }^{\mathrm{m}} 02$ were automatically excluded from the data set. These observations have been earlier published in Paper III.

\section{The January 1998 temperature map}

In Paper II, we found that during the years 1994-1997 the active region in FK Com was shifting $0.22 \pm 0.03$ in phase within a year. This phase shift was most likely caused by a difference between the used photometric period 2 d 4002466 (from 25 year observations, Jetsu et al. 1993) and the real spot rotation period for this time, 2. 4037 (Paper II). The new map for January 1998 was first calculated with the old ephemeris given by Jetsu et al. (1993): 2439252.895 + 2 d $4002466 E$. Then, cross-correlation between the maps for June 1997 and January 1998 revealed that the rate of $0.22 \pm 0.03$ phase per year is still valid for the autumn 1997 . To exclude the phase shift due to the period differences, the period 2. 4037 was used in this paper.

The inversions were done for all the lines in the spectral region between $6416 \AA$ and $6444 \AA$ using the Tikhonov Regularization method code, INVERS7, written by N. Piskunov and modified by T. Hackman (Piskunov 1991; Hackman et al. 2001). The stellar parameters adopted for surface imaging can be found in Table 2. More details on the method and the selection of the stellar parameters are given in Papers I and II. The model spectrum used in these calculations is the same as earlier used for the years 1996-1997 (Paper II).

The surface image and the fits to the observations are shown in Fig. 1. The mean deviation of the spectroscopic observations from the model is $0.458 \%$, which corresponds 

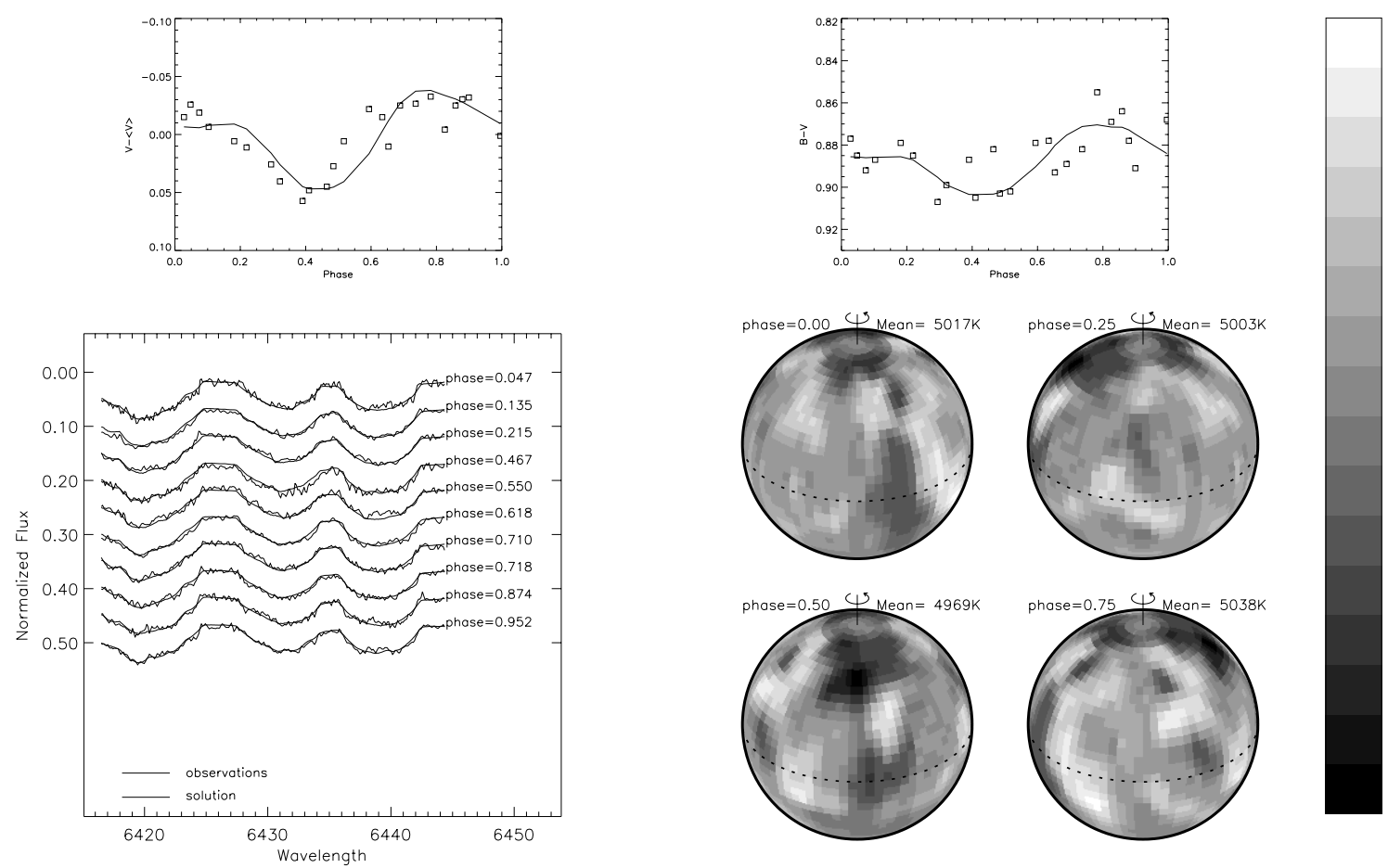

$5450 \mathrm{~K}$

$5380 \mathrm{~K}$

$5310 \mathrm{~K}$

$5240 \mathrm{~K}$

$5170 \mathrm{~K}$

Fig. 1. The surface temperature map of FK Com for December 1997-January 1998 obtained with the Tikhonov regularization from the $6416-6444 \AA$ region with $v \sin i=155 \mathrm{~km} \mathrm{~s}^{-1}$. A grid of 40 latitudes and 80 longitudes across the stellar surface is used in the map. Calculated and observed spectral lines are shown by thick and thin lines, respectively. Photometric observations, from which the mean magnitude $(\langle V\rangle)$ has been subtracted, are plotted with squares, and curves calculated from the map are presented by lines.

Table 2. Adopted values of the stellar parameters for surface imaging.

\begin{tabular}{ll}
\hline Parameter & Adopted value \\
\hline$T_{\text {eff }}$ (unspotted) & $5025 \mathrm{~K}$ \\
$\log g$ & 3.5 \\
Period & 2.4037 \\
$v \sin i$ & $155 \mathrm{~km} \mathrm{~s}^{-1}$ \\
Inclination & $60^{\circ}$ \\
Microturbulence & $1.0 \mathrm{~km} \mathrm{~s}^{-1}$ \\
Macroturbulence & $2.0 \mathrm{~km} \mathrm{~s}^{-1}$ \\
\hline
\end{tabular}

to an average $S / N$ ratio of 218 . The main feature is an extended cool area centered on phases $0.4-0.6$ in the latitude interval $45^{\circ}-72^{\circ}$. This feature has a temperature of $\sim 600 \mathrm{~K}$ below the unspotted surface. Another extended cool area with a temperature of $\sim 500 \mathrm{~K}$ below the unspotted temperature can be seen around the phases $0.8-1.1$ at approximately the same latitudes as the other cool area.

Photometric observations and the photometry calculated from the map are also shown in Fig. 1. Note that they have not been used in the inversions as a constrain. As can be seen, the $B-V$ colours and the $V$ magnitudes calculated from the map fit the simultaneous photometric observations well. When looking at the $V$ magnitude, the calculated curve seems to be very slightly shifted towards larger phases in comparison to the observations. Our photometry from the beginning of 1998 shows that the weaker active region at the phase $0.8-1.1$ evolves rapidly during
January-March and becomes again stronger than it was in late 1997. The small shift in phase of the minimum of the calculated $V$ magnitudes can most likely be explained by the fact that the spectroscopic observations were obtained over a time period of 17 nights, covering 7.1 rotations, during a time of a rapid light curve evolution. Due to the reasonably good reproduction of the shape and position of the light curve minimum, the position and structure of the main features in the map are considered to be reliable.

\section{The new "flip-flop"}

The photometric observations from the 1990's (Papers III and IV) show that the "flip-flops" have occurred during the summer 1994 and again during autumn 1997, in accordance with the 3.3-year cycle. In Fig. 2 the photometric observations for 1997 and beginning of 1998 are shown together with the spot filling factor maps obtained by inversions from the light curves. The light curve inversions are done using a technique similar to that used by Rodonó et al. (2000) and explained in more detail in Paper IV where the results of inversions for 35 years of photometric observations of FK Com are presented.

Figure 2 clearly shows the "flip-flop" that occurred during the autumn 1997. In the second half of 1997, the old active region around the phase 1.0 has significantly diminished, while the new one near the phase 0.5 has appeared. In the beginning of 1998, the old active region becomes again rapidly stronger than it was during December 1997. 


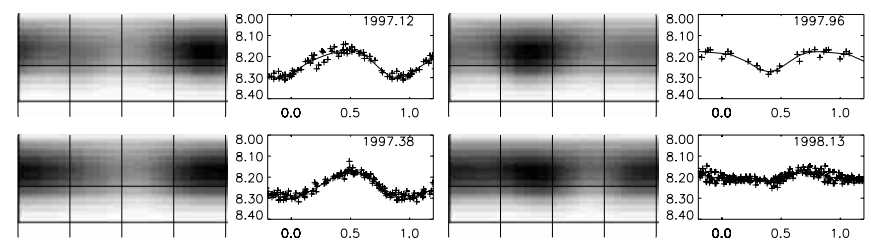

Fig. 2. The results of the light curve inversions for the year 1997 and beginning of the year 1998. The darker regions indicate larger spot filling factor. The grid in the maps indicates the equator and 4 longitudes separated by $90^{\circ}$. In the photometry the observations are presented by crosses and the calculated curve with a line.
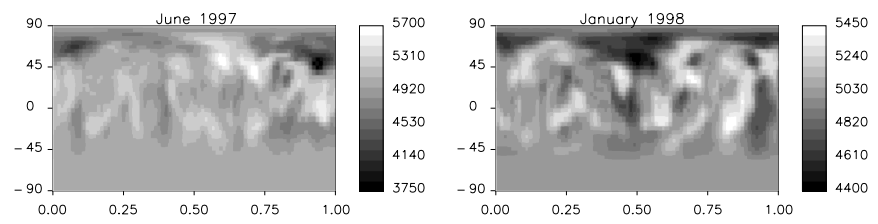

Fig. 3. The surface temperature maps for June 1997 and January 1998. The grey scale gives the temperatures in Kelvin.

The photometry for 1998-1999 (Papers III and IV) shows that from January 1998 till June 1999 both active regions are simultaneously active, resulting in a very flat and featureless light curve.

Figure 3 shows the temperature maps obtained with surface imaging for June 1997 and January 1998. In June 1997, the spots are concentrated around the phase 1.0 and there is no evidence of spots at the phase 0.5 . In January 1998, the most contrast spot group is seen at the phase 0.5 and a secondary group is around the phase 1.0. It is reasonable to assume that the spot group at the phase 1.0 during January 1998 is the same as the one in the June 1997 map at the same phase, the activity of the spot group has just diminished. The spot group, 0.5 in phase apart from the old active region, is a new active region which has appeared on the other side of the star, as predicted in the "flip-flop" phenomenon. The cross-correlation of the maps, done as described in Paper II, using latitudes 42.7583.25 , yields phase shifts of -0.04 and 0.50 for the old and new active regions, respectively.

In FK Com, usually only one of the active longitudes is active at a time, as can be seen, for example, from the temperature maps for the years 1995-1997 (Papers I and II). Anyhow, near the "flip-flop" both active longitudes become simultaneously active, like in summer 1994 (Paper I) and in 1997-1998 (Fig. 2), and the change of the relative strengths of the active regions causes the "flip-flop".

\section{Conclusions}

With the Doppler imaging technique and light curve inversions we showed clearly that FK Com possesses the same spot activity pattern as observed in binary components of the RS CVn type and in a young dwarf LQ Hya, namely the "flip-flop" phenomenon caused by changing the relative strengths of the spot groups at the two active longitudes.
The fact that different types of rapidly rotating stars show the same phenomenon in detail supports the idea that it is the rapid rotation that determines the principal component of the stellar activity, regardless of the origin of the rapid rotation.

Since during the "flip-flop" the light curve undergoes rapid and irregular changes, one should be careful in interpreting such changes as spot period variations and, as a consequence, the stellar differential rotation.

Acknowledgements. The work of H.K. was supported by the Finnish graduate school in Astronomy and Space Physics and Vilho, Yrjö and Kalle Väisälä Foundation. K.G.S. acknowledges the receipt of FWF grant S7301 from the Austrian Science Foundation.

\section{References}

Berdyugina, S. V., \& Tuominen I. 1998, A\&A, 336, L25

Berdyugina, S. V., Berdyugin, A. V., Ilyin, I., \& Tuominen, I. 1998, A\&A, 340, 437

Berdyugina, S. V., Berdyugin, A. V., Ilyin, I., \& Tuominen, I. 1999, A\&A, 350, 626

Berdyugina, S. V., Berdyugin, A. V., Ilyin, I., \& Tuominen, I. 2000, A\&A, 360, 272

Berdyugina, S. V., \& Tuominen, I. 2001, A\&A, submitted

Bopp, B. W., \& Stencel, R. E. 1981, ApJ, 247, L131

Chugainov, P. F. 1966, Inf. Bull. Var. Stars, No. 172

Hackman, T., Jetsu, L., \& Tuominen, I. 2001, A\&A, 374, 171

Hall, D. S. 1991, in The Sun and Cool Stars: activity, magnetism, dynamos, Proc. IAU Coll. 130, ed. I. Tuominen, D. Moss, \& G. Rüdiger (Springer, Heidelberg), 380, 353

Henry, G. W., Eaton, J. A., Hamer, J., \& Hall, D. S. 1995, ApJS, 97, 513

Jetsu, L., Pelt, J., Tuominen, I., \& Nations, H. L. 1991, in The Sun and Cool Stars: activity, magnetism, dynamos, Proc. IAU Coll. 130, ed. I. Tuominen, D. Moss, \& G. Rüdiger (Springer, Heidelberg), 381

Jetsu, L., Pelt, J., \& Tuominen, I. 1993, A\&A, 278, 449

Jetsu, L., Tuominen, I., Grankin, K. N., Mel'nikov, S. Yu., \& Shevchenko, V. S. 1994, A\&A, 282, L9

Jetsu, L. 1996, A\&A, 314, 153

Korhonen, H., Berdyugina, S. V., Hackman, T., et al. 1999, A\&A, 346, 101, Paper I

Korhonen, H., Berdyugina, S. V., Hackman, T., Strassmeier, K. G., \& Tuominen, I. 2000, A\&A, 360, 1067, Paper II

Korhonen, H., Berdyugina, S. V., Tuominen, I., et al. 2001a, A\&A, 374, 1049, Paper III

Korhonen, H., Berdyugina, S. V., Tuominen, I., et al. 2001b, A\&A, submitted, Paper IV

Piskunov, N. E. 1991, in The Sun and Cool Stars: activity, magnetism, dynamos, Proc. IAU Coll. 130, ed. I. Tuominen, D. Moss, G. Rüdiger (Springer, Heidelberg), 309

Ramsey, L. W., Nations, H. L., \& Barden, S. C. 1981, ApJ, 251, 101

Rodonó, M., Messina, S., Lanza, A. F., Cutispoto, G., \& Teriaca, L. 2000, A\&A, 358, 624

Strassmeier, K. G. 2001, In The 11th Cambridge Workshop on Cool Stars, Stellar Systems and the Sun, ed. R. J. García López, R. Rebolo, \& M. R. Zapatero Osorio, ASP Conf. Ser., 223, 271

Walter, F. M. 1981, ApJ, 245, 677 